\title{
Measuring the Energy Resolution of MicroBooNE at the MeV-Scale
}

\section{Elise Chavez • GEM • University of Wisconsin-Madison • Advisors: Joseph Zennamo and Fernanda Psihas (Fermilab)}

\section{I - Introduction}

MicroBooNE has the opportunity to study the energy resolution of a LArTPC (Liquid Argon Time Projection Chamber) at low MeV energies through doping the argon with radon222. Here we looked at MicroBooNE simulation to examine the energy resolution for the collection plane (plane 2) using the reconstructed energy of a $\beta$ that comes from a bismuth 214 - polonium 214 coincidence decay in the radon chain.

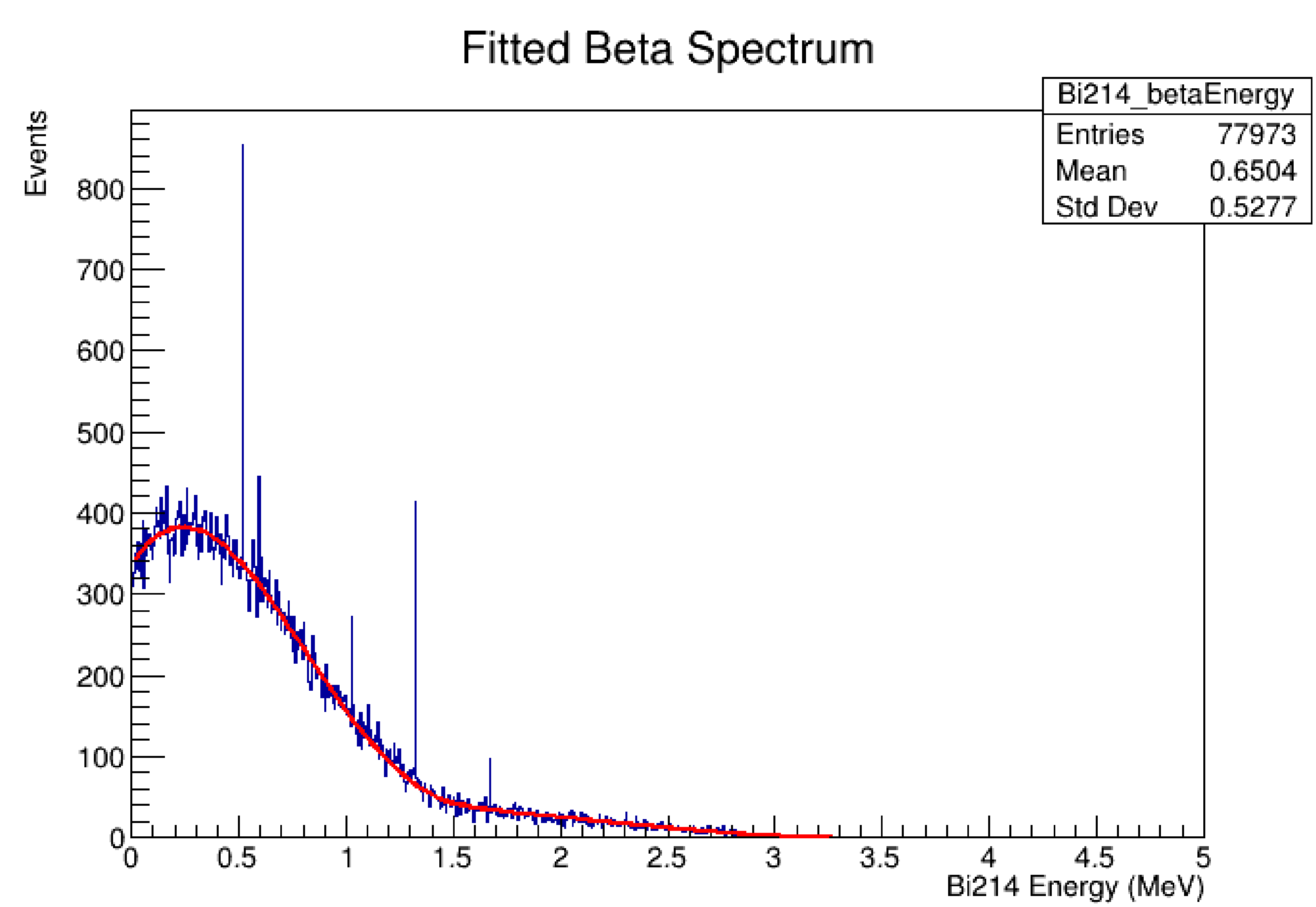

Figure 1: In progress. The fitted $\beta$ spectrum produced from a Bi214 decay. This is the simulated true energy fitted with Betashape from the International Atomic Energy Agency (IEAE) Nuclear Data Services (NDS) Live Nuclide Chart. (https://nds.iaea.org/relnsd/vcharthtml/VChartHTML.html)

\section{II - Purpose}

To measure the energy resolution (precision) of MicroBooNE at low MeV energies using a bismuth 214 - polonium 214 coincidence decay in simulation

\section{III - Methods}

Once simulated, we defined fractional energy reconstruction error to be $\left(E_{\text {Reconstructed }}-E_{\text {True }}\right) / E_{\text {True }}$ and plotted this against reconstructed energy. Then we went bin by bin in this space and fitted each with gaussians.

Fractional Energy Reconstruction Error Vs. Reconstructed Energy

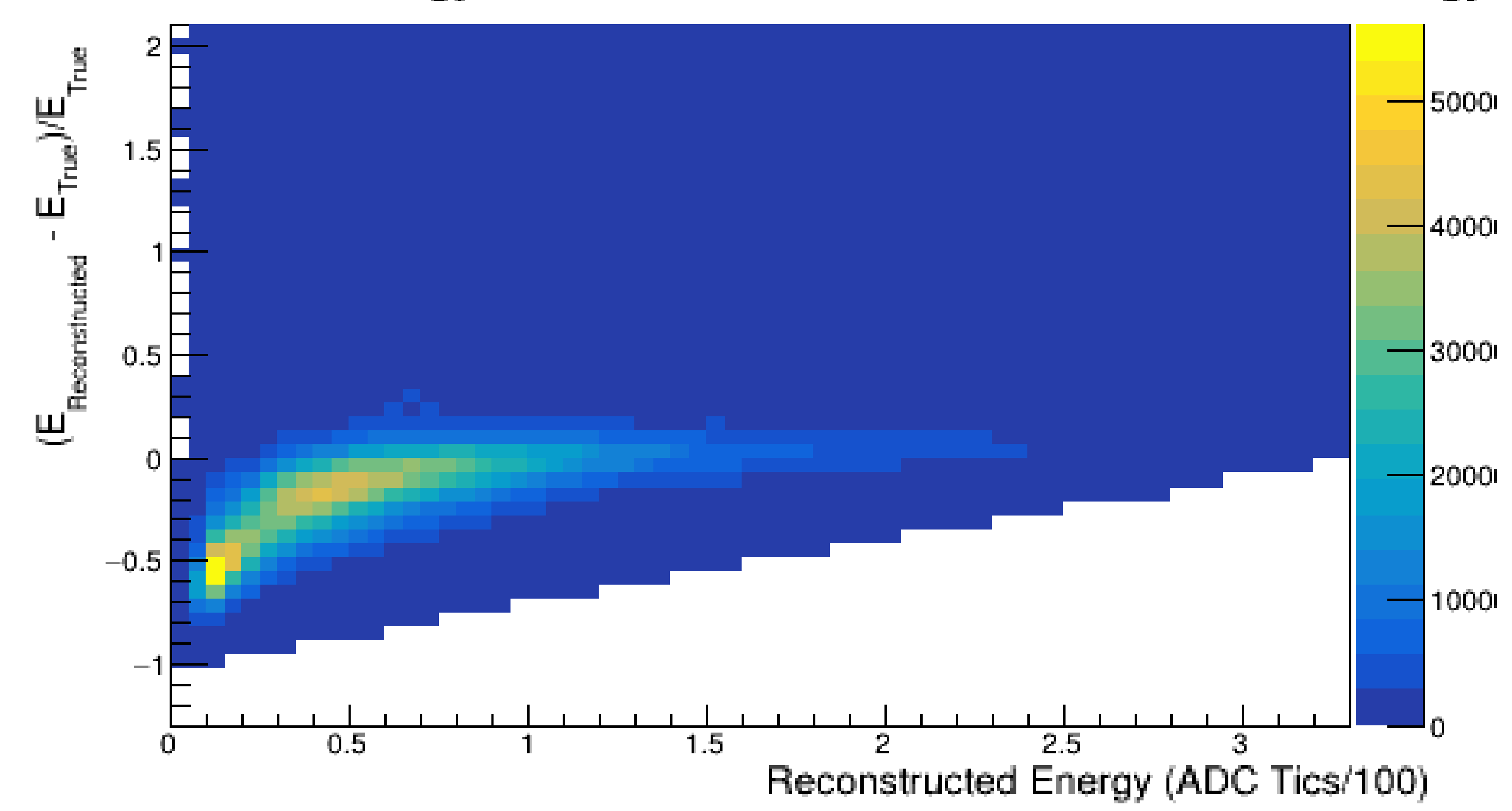

Figure 2: In progress. Fractional Energy Reconstruction Error vs. Reconstructed Energy for the decay $\beta$ with the $x$ axis being a rough conversion to $\mathrm{MeV}$.

Collection Plane Fractional Energy Reconstruction Error for $1.9 \mathrm{MeV}<\mathrm{E}_{-}$Reco $<2.25 \mathrm{MeV}$

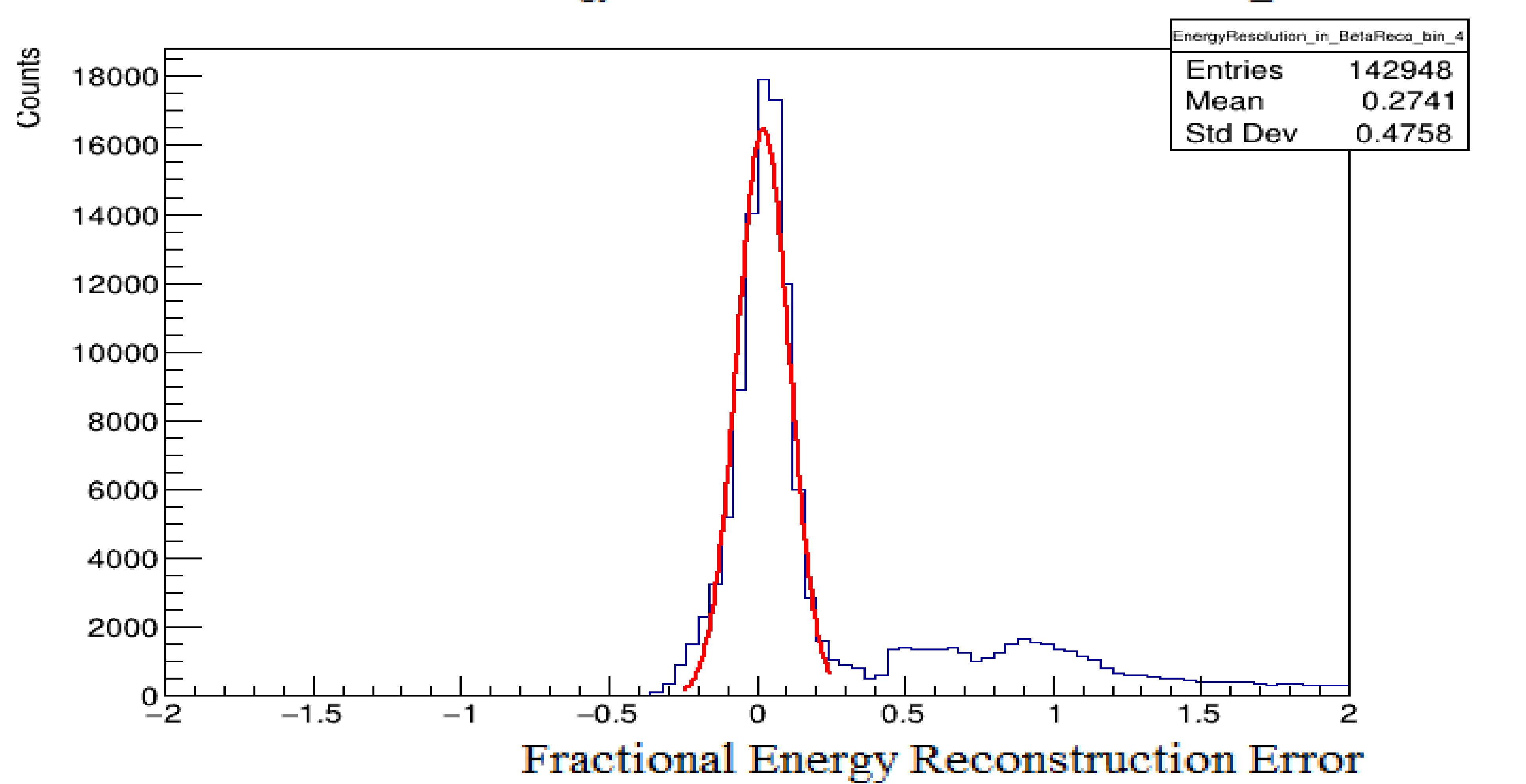

Figure 3: In progress. Gaussian fit to collection plane fractional energy reconstruction error histogram for $1.9 \mathrm{MeV}<\mathrm{E}$ Reco $<2.25 \mathrm{MeV}$.

\section{IV - Results}

From the fitted plots, we extracted the widths of the of the gaussian fits which were then plotted for each bin spanning the space. This width represents the energy resolution. This gave us a plot we can fit and use to measure the energy resolution of real data.

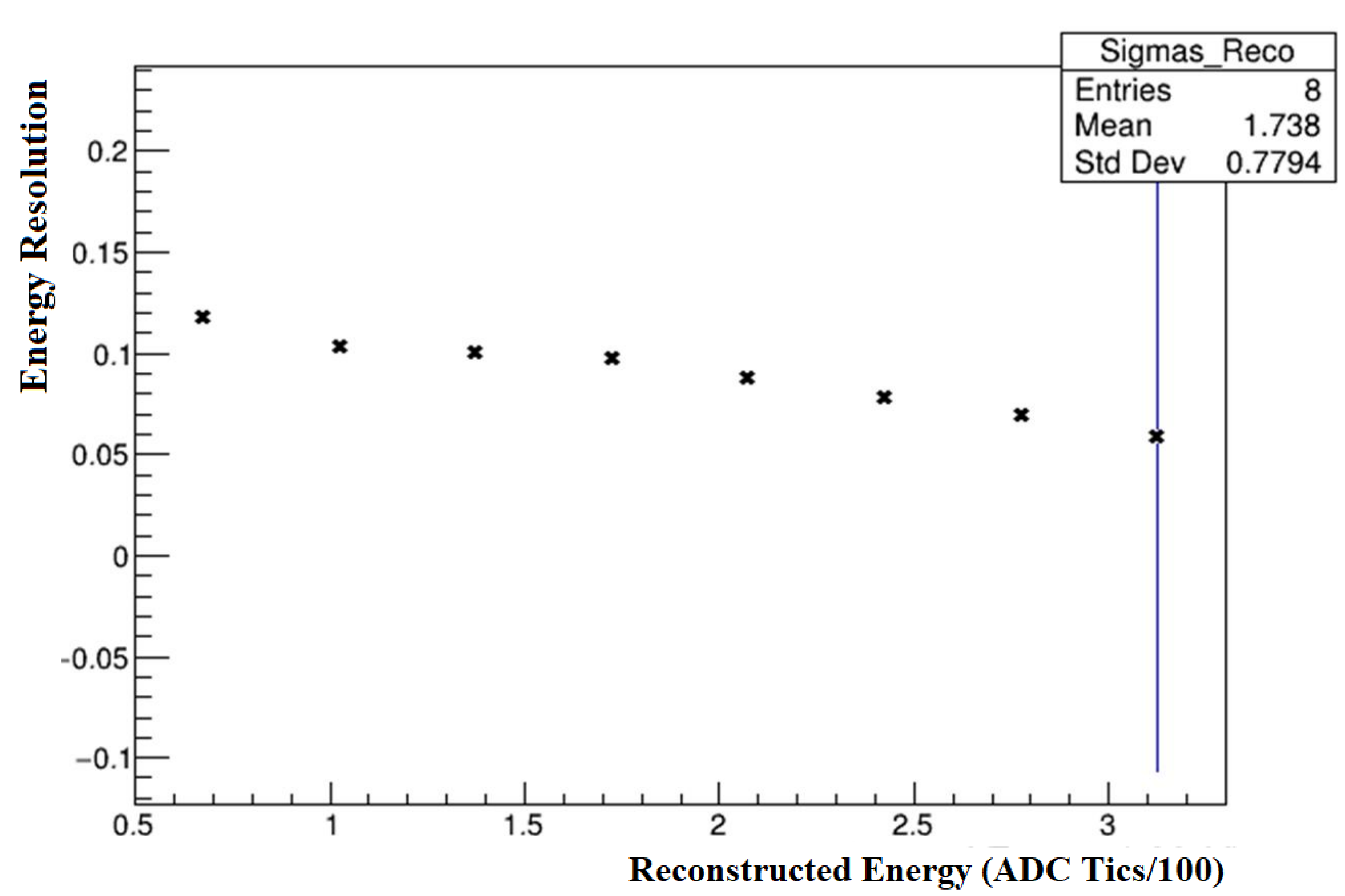

Figure 4: In Progress. The width of gaussian fits to the collection plane fractional energy reconstruction error histograms for each bin of reconstructed energy.

\section{V - Conclusions}

We have created a functional description of the bismuth-214 spectrum in Figure 1 and studied how this function description changes with reconstructed energy. This can then be used to measure the energy resolution of real data.

\section{$\mu B O O N E$}

$\mu B O O N<$ 